\title{
Contrast restoration of road images taken in foggy weather
}

\author{
Houssam Halmaoui $\quad$ Aurélien Cord \\ UniverSud, LIVIC, Ifsttar \\ 78000 Versailles \\ Houssam. Halmaouidifsttar.fr \\ Aurélien.Cordeifsttar.fr
}

\author{
Nicolas Hautière \\ Université Paris-Est, LEPSIS, Ifsttar \\ 75015 Paris \\ Nicolas.Hautièredifsttar.fr
}

\begin{abstract}
Driver assistance systems based on camera are strongly disturbed by the presence of foggy weather. The restoration of images, as pre-processing, would improve the performances of such systems. In this paper, we propose a method to restore the image contrast of foggy road scenes combining a physical approach, based on Koschmieders model and a signals approach, based on local histogram equalization. Then we optimize the parameters of our method using a simulated annealing. This method, evaluated on a reference image database, presents a significant improvement compared to other methods and gives consistent results for both homogeneous and inhomogeneous fog.
\end{abstract}

\section{Introduction}

Recent developments in image processing and electronics induced a strong increase of the number of Advanced Driver Assistance Systems (ADAS) based on on-board cameras which equip recent vehicles. Their applications are numerous: detection and recognition of road signs, lane markings and obstacles. In the presence of fog, the road visibility distance decreases considerably, which increases the risk of accidents. Similarly, because of degraded visibility conditions, ADAS based on camera could give erroneous results. It may generate false alarm to the driver or misinterpret a dangerous situation.

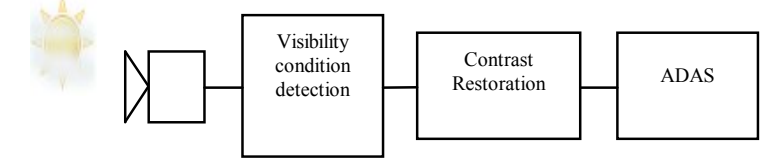

Figure 1: Global scheme of proposed approach.

In this work, we focus on images degraded by foggy conditions during day time. During the last 10 years, several methods have dealt with this subject. In this paper, we propose a two-stage procedure, presented in Figure 1: visibility condition detection then contrast restoration.
The detection is done using the approach proposed by Hautière et al. [4].

The contrast restoration stage aims to provide for the ADAS an improved image where the impact of the fog is strongly diminished.

Contrast restoration in real time allows extending the operating range of current vision systems and thereby potentially reducing the number of accidents. It also meets the economic dimension which is to reduce the cost of embedded systems by integrating several functions into a single sensor.

Contrast enhancement techniques are numerous $[5,6$, 16]. However, the effect of the fog reduces the contrast of observed objects as a function of the distance to the camera. Thus, conventional techniques do not provide satisfactory restorations, because they cannot take into account distance variations existing in the scene. More recently, methods dealing with foggy image restoration rely on the physical model of Koschmieder [9]. This model establishes a relationship between apparent luminance and intrinsic luminance of an object in a scene with fog. This model has four unknowns: the intrinsic luminance, the sky luminance, the fog density and the object-observer distance. Therefore, we have a single equation with four unknowns; the problem is ill-posed. In order to overcome this problem, [7] and [11] use approximate 3D model. [10] uses several images taken under different visibility conditions. Some approaches make assumptions about the scene: constraints on image hue [14], on transmission [1], on image statistics [2]. In the state of art, other methods are dealing with dehazing $[8,12,13]$. However, they are generally not suitable to ADAS images [15] for two reasons: they are not real time or they do not take into account the context of the road (road surface, lane marking, and road signs).

Relying on the method proposed by [4] to segment road surface vertical objects, we present in this paper, a new approach for the restoration of vertical objects. It combines a physical approach, based on Koschmieders 
model and a signal based approach, relying on local histogram equalization.

The article is organized as follows. In section 2 , the physical model of fog is introduced. In section 3, we present the different steps of our contrast restoration method: detection of vertical objects, evaluation of their intrinsic luminance by local histogram equalization and estimation of the scene depth map. In section 4 , the parameters are optimized and their sensitivities are studied. In section 5 , we evaluate the performance on an available image database in comparison with other methods.

\section{Physical model of fog}

The fog is constituted of small water droplets floating in the air that scatters the light: Koschmieder's Law [9]. During day time, two effects are combined: (1) the intrinsic luminance of an object decreases exponentially with the distance to the observer, (2) the contribution of the atmospheric luminance increases exponentially with distance:

$$
I=I_{\mathrm{o}} e^{-\beta d}+I_{s}\left(1-e^{-\beta d}\right)
$$

With I the apparent luminance of an object, $I_{0}$ its intrinsic luminance, $I_{s}$ the atmospheric luminance which correspond to the luminance of the sky, $d$ the distance between the object and the observer and $\beta$ the extinction coefficient of the atmosphere. Assuming that the camera has a linear response, we can apply the equation (1) directly to pixel's intensity.

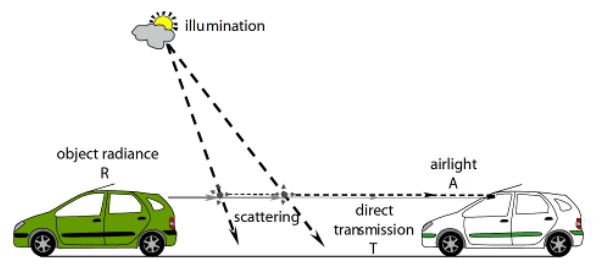

Figure 2: Scattering of light by daytime fog.

The Figure 2 illustrates the principles of light scattering by daytime fog. The radiance $R$ corresponds to $I_{0}$, the direct transmission is the first term of the equation (1) and the airlight is the second tem of this equation.
The restoration formula is given by inverting this model:

$$
I_{\mathrm{o}}=I e^{\beta d}+I_{s}\left(1-e^{\beta d}\right)
$$

This model does not apply to daytime scenes containing any bright object, because it does not take into account the artificial light and the halo effect.

\section{Method}

Figure 3 shows the different steps of our algorithm described in this section.

\subsection{Fog characterization and vertical objects segmentation}

The method introduced by [4] allows both the fog characterization and the vertical objects segmentation. First the extinction coefficient $\beta$ is estimated by using equation (1) and searching the inflection point on the intensity with respect to the image line [4]. The depth map of the road surface, assumed flat, is estimated using the camera parameters. In this approximate depth map, the distance of vertical objects is overestimated.

The equation (2) shows that, due to this overestimation, the estimated intensity $I_{o}$ tends towards zero exponentially. Thus, it allows the segmentation between road plane and vertical objects.



Figure 4: Original image, restoration using planar assumption and segmentation result.

Figure 4 shows an example of restoration and segmentation. The road surface is nicely restored using this approach. In the rest of the paper, we will focus on restoration of objects above the road plane.

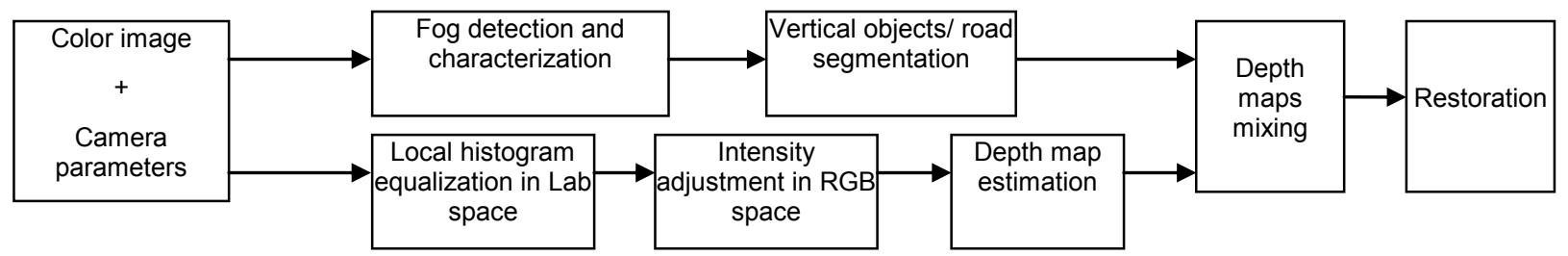

Figure 3: Different steps of our approach. 


\subsection{Histogram equalization}

The local histogram equalization is used to provide a first estimation of the intrinsic luminance image. It enhances contrast locally and uniformly across the entire image. Thus it mitigates the fog effect by making all details in the image visible (see Figure 5.b).

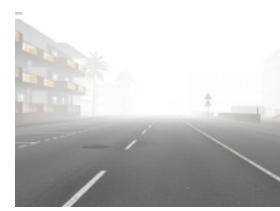

(a)

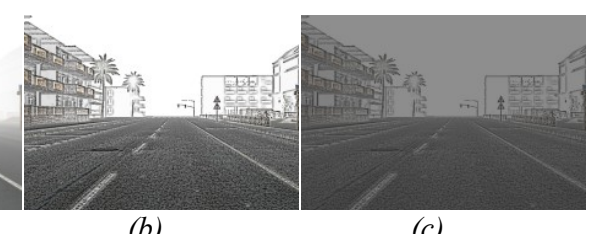

(b) (c)
Figure 5: original image (a), equalized image (b) and image after intensity adjustment (c).

Histogram equalization distributes the intensities of pixels over the entire range of the histogram or on a certain interval, thereby increasing the contrast of the image. Figure 6 illustrates its principle: first the normalized intensity distribution function is calculated, and then applied on the image histogram in order to spread it uniformly.
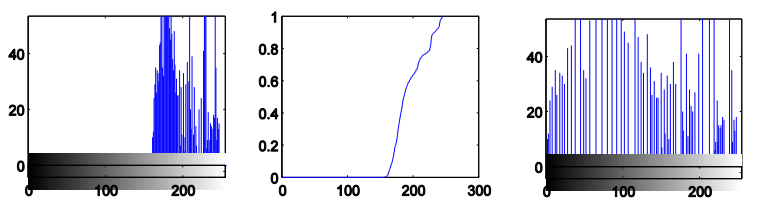

Figure 6: Original histogram, normalized intensity distribution function and histogram equalized uniformly.

In case of foggy images, the local histogram equalization is done on a partition of the image into small windows of size $3 * 3$. For each window, the eight contiguous blocks are taken into account to calculate the function. It reduces the final difference in intensity between neighboring blocks in the restored image. This operation is then performed on 81 pixels ( 9 blocks) and only the central block is modified. We apply the additional constraint $I_{0}<I$ resulting from equation (1 with the additional constrain that the sky is the brightest area in outdoor scenes $\left(\mathrm{I}_{0}<\mathrm{I}_{\mathrm{s}}\right)$. Therefore we apply equalization between 0 and the maximum of intensity for the central block.

When the area to process is uniform, the equalization does not modify this area (such areas have no interest because they are containing no information).

On the contrary, when we have areas with texture, the equalization will depend on neighboring blocks: two blocks having the same texture but different contiguous blocks will not be identically restored because the shapes of their normalized intensity distribution function are different. This generates blocks effects that are attenuated in the following (Section 3.5) by the smoothing of the depth map.

To reduce the processing time, color images are converted in Lab color space and the equalization is performed only on the luminance component (one channel instead of three). Indeed, the equalization step is the most time consuming in the overall process.

\subsection{Intensity adjustment}

Histogram equalization restores details that were barely visible in the foggy image. However, it strongly modifies the image dynamic range, providing intensity values from 0 to the maximum of $I$. Therefore, the dynamic is adjusted by a simple affine transformation " $a * I_{\text {equalized }}+b$ ". The constants $a$ and $b$ are optimally chosen $(a=0.2$ and $b=$ 40) using simulated annealing (see Section 4.2). Figure 5 shows the equalized images before and after dynamic range adjustment.

\subsection{Approximate depth map}

From equation (1), we derive an expression for the distance of each pixel depending on images $I$ and $I_{0}$ with and without fog, the intensity of the sky $I_{s}$ and the $\beta$ coefficient:

$$
d=-\frac{1}{\beta} \ln \left(\frac{I-I_{s}}{I_{0}-I_{s}}\right)
$$

By replacing, in equation (3), $I_{0}$ by the result of the histogram equalization, an approximate depth map of the scene is obtained (Figure 7).

Note also that the estimation of $\beta$ is only required to perform the restoration of the road plan. To restore vertical objects, instead of estimating a depth map, we could estimate a $t$ map, with $t=\beta * d$.

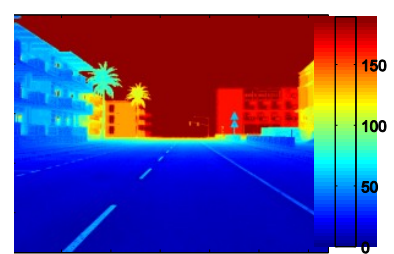

Figure 7: Estimated depth map (m).

If the planar assumption method does not provide a good estimation of the coefficient $\beta$, we can apply our 
algorithm on the entire image. This may occur when there are many obstacles on the road.

In the case of color images, we apply the equation (3) on each channel, obtain three depth maps and choose $d$ as the minimum of the three maps.

\subsection{Smoothing}

The obtained depth map is affected by block effect due to the local equalization (Figure 7). This is attenuated by an adapted smoothing filter.

A Gaussian filter with a large size compared to the window equalization, here $11 * 11$, is applied to homogenize the depth of large objects. However, around objects borders, depth values are false: depth of the most distant objects is spread inside of closest object and vice versa. It generates dark (respectively clear) area in restored image according to equation (2) (see Figure 8.a). Applying a Sobel filter to the restored image allows us to detect the gradient maxima that correspond to boundaries between objects. By dilating these edges with half of the Gaussian size, we detect area where the depth is misestimated (see Figure 8.b). We apply a second smoothing using a Gaussian filter of size $3 * 3$. The final depth map for pixels in this area is a combination of both filtered depth maps:

$$
d=\delta * d 1+(1-\delta) * d 2
$$

With $\delta$ the normalized distance between pixel and the edges, $d 1$ the depth map obtained with the Gaussian filter of size $G S 1=11$ and $d 2$ the depth map obtained with the Gaussian filter of size $G S 2=3$.

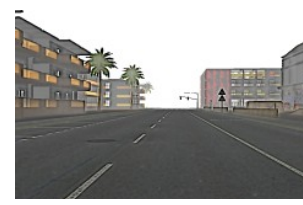

(a)

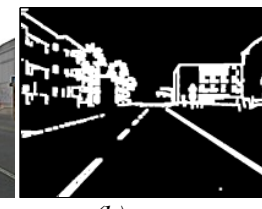

(b)

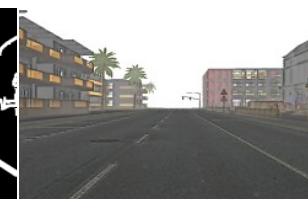

(c)
Figure 8: restoration using $d 1$, segmentation of misestimated area, final restoration using $d$.

Finally, the restoration is performed using this depth map with the equation (2) (Figure 8.c).

\subsection{Depth map mixing}

The depth map could be mixed with the depth map of the road surface, assumed flat, evaluated in a previous step (Section 3.1). In this case, we apply the distance $d$ only for vertical object, segmented during the same step.

The final restoration using this mixed depth map is called "with planar assumption" in the following.

\section{Parameter optimization and sensitivity study}

\subsection{Database}

Since it is difficult to obtain the same road image with and without fog, a database of synthetic images containing 66 color images of different scenes (city, highway, rural) was produced by [15]. Thanks to the access to depth maps, using the Koschmieders model four different types of fog were added to images: uniform, variable sky intensity, variable fog density and variable sky intensity and fog density.

Note that for the optimization of parameters and the sensitivity study, only uniform fog images are used. However the whole database is used to compare our approach with other restoration methods in section 5.2.

The metric used to calculate the error is the sum of absolute differences between pixel values of the restored image and the reference image without fog, without taking into account the sky to avoid biased results.

Let's recall the parameters of our algorithm: $W S$ the window size of equalization, $a$ and $b$ the coefficients allowing the intensity adjustment of the equalized image and $G S 1$ and $G S 2$ the size of the large and small Gaussian filters, respectively. GS2 size is fixed to 3 in order to recover the small details.

\subsection{Simulated annealing}

In order to find the best combination of parameters, we have extracted, from the database, 5 representative images with uniform fog. On these 5 images, we have applied a simulated annealing.

The simulated annealing is an iterative optimization algorithm for finding the global minimum of a function. In our case we seek to minimize the average error between the 5 restored images and the corresponding original images without fog.

The principle is the following: at each iteration a new set of parameters is randomly generated in the neighborhood of the last selected parameters. This set is used to perform restoration and the error is compared to the best error previously found. The set is selected in two cases: (1) if the error decreases. (2) To avoid that the algorithm converges to a local minimum, larger errors are accepted in a random manner, according to a variable called "temperature" that decreases at each iteration (cooling cycles): the greater is the error the lesser the set are selected. Then it reiterates. Finally, if the algorithm 
keeps the same parameter set after several consecutive iterations it is stopped. By running simulated annealing with our algorithm, we get the best score for the parameters presented in table I.

\begin{tabular}{|c|c|c|c|c|}
\hline Parameter & $W S$ & $G S 1$ & $a$ & $b$ \\
\hline Value & 5 & 15 & 0.2 & 40 \\
\hline Std & 0.55 & 0.51 & 6.22 & 2.32 \\
\hline
\end{tabular}

Table I: Best parameters determined by simulated annealing. Std corresponds to error standard deviation among the 66 images.

The values of WS and GS1 to which the simulated annealing converges, may be explained by the following remarks. The distant objects are strongly affected by the fog. Because their pixel size is small, a small WS size leads to a better restoration of their contrast. Similarly, the details of near objects (such as road signs) will be better restored with a small WS. The parameter GS1 must be larger than WS in order to achieve the best homogenization of the depth map for large object.

\subsection{Sensitivity study}

To perform the sensitivity study, one of the parameters varies when others are fixed. The average error is calculated over all 66 images with uniform fog from the synthetic database. The results are shown in figures 9 and 10.

We observe in Figure 9 that the method is not very sensitive to changes in WS and GS1 (the error is in the range 22 to 24). We have set the values of these parameters to $W S=5$ and $G S I=11$. On the contrary, Figure 10 shows that it is very sensitive to changes on coefficients $a$ and $b$. Values of $a=0.2$ and $b=40$ give the best results.

As explained above, the method without "planar assumption" is insensitive to $\beta$, because it only depends on the product $\beta^{*} d$. Thus, we studied the sensitivity of $\beta$ for the method with "planar assumption". As shown in [4] the parameter $\beta$ is inversely proportional to the distance of visibility Vis, we chose to work on visibility rather than $\beta$. In Figure 11, we have plotted the error according to Vis. This curve presents a minimum at $80 \mathrm{~m}$., which corresponds to the chosen visibility in synthetic images. We observe that the method is quite sensitive to changes in Vis.

For very small values of Vis (less than 40m.), the error decreases. This comes from the fact that the road surface restoration is very sensitive to Vis. However, when decreasing $V i s$, a larger part of the image is classified as vertical object during the segmentation step. Thus, a smaller part of the image is restored using the wrong estimated value of Vis. The rest of the scene is restored as vertical objects and is less penalizing by an incorrect value of Vis, because it is compensated by an incorrect, but proportional, estimation of the depth.
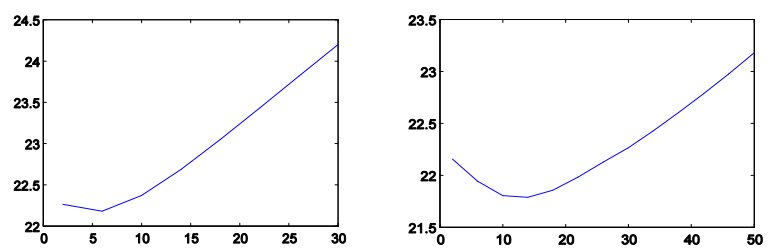

Figure 9: Sensitivity to WS and GS1.
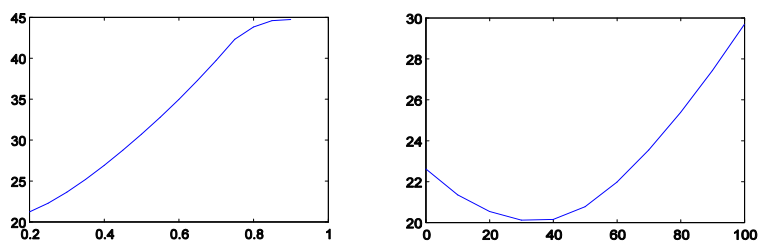

Figure 10: Sensitivity to a and $b$.



Figure 11: Sensitivity to visibility.

\section{Results}

\subsection{Qualitative results on synthetic and real images}

The Figure 12 shows the restoration results on synthetic images using the presented method with the planar assumption.

We see that using our approach without planar assumption (2nd column in Figure 12), the lane markings are over-restored. This is due to the fact that the lane markings have a white color. Thus the equalization operation increases their contrast as if they were distant objects. To circumvent this problem, the combination with the planar assumption (3rd column in Figure 12) produces a very nice restoration of these areas. It emphasizes the interest of the combination with planar assumption on typical road images. 

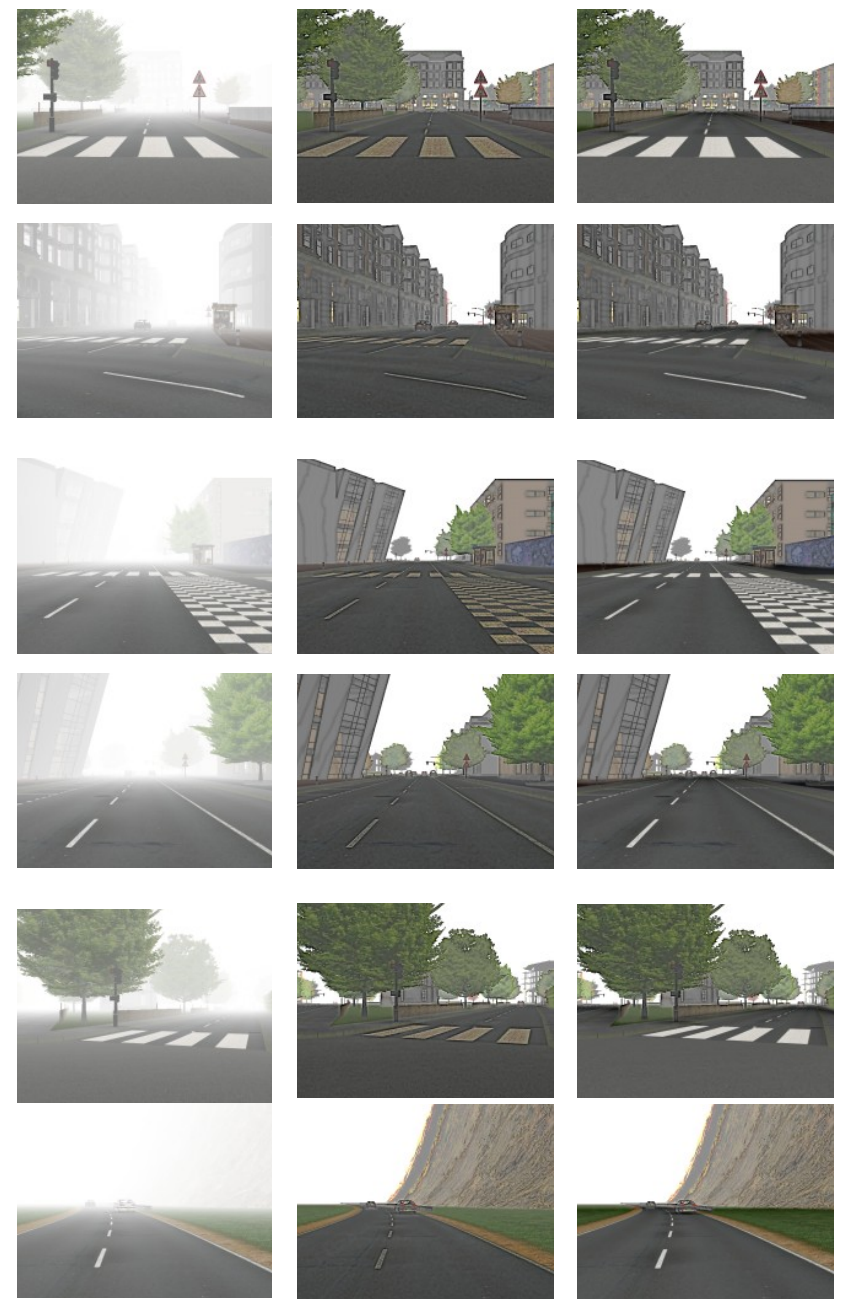

Figure 11: Results of restoration on synthetic images. Original image, restoration using our method and restoration using the combination with planar assumption.

Other applications where we do not have access to camera settings are presented in Figure 13 and 14. Our approach without planar assumption still provides good results, for both grayscale and color images. It shows that the contrast restoration is increasing details that are barely visible in foggy images.

Fig 14 displays some unrealistic colors. They results from the atmospheric light $I s$ of the observed scenes that is not white (contrary to the synthetic image where $I_{s}$ equals to 255 on each RGB channels). A solution could be to operate a white balance on input images, in order to obtain a white sky. However, as our method is focused on ADAS, the objective is not to obtain images with the best visual appearance but rather to provide ADAS with an image containing as much information as possible.
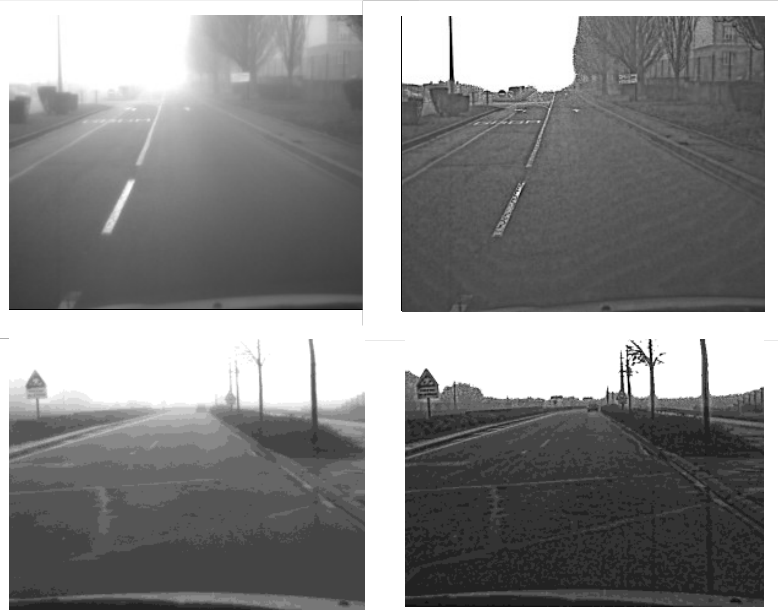

Figure 13: Results of restoration on real grayscale images.
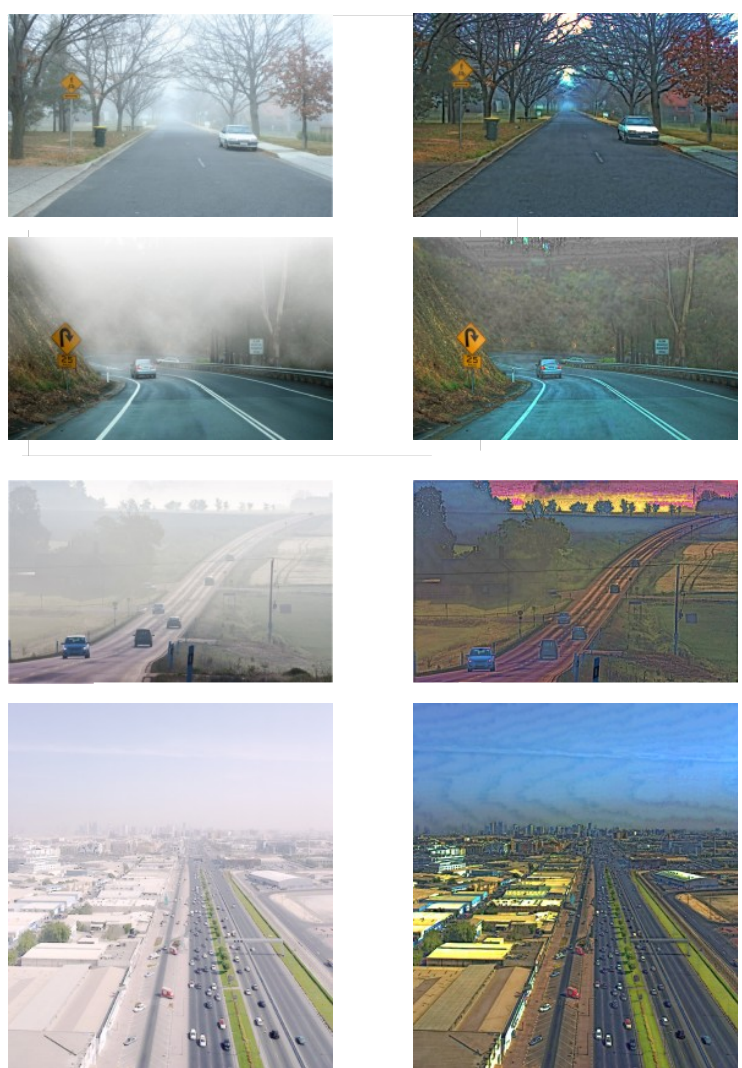

Figure 14: Results of restoration on real color images without planar assumption.

\subsection{Quantitative results on synthetic images}

In order to evaluate our results, we compare the restoration errors obtained by other approaches on the synthetic database (cf. Section 4.1). This evaluation is presented in table II, for the 4 types of fog. The error 
values of other approaches were extracted from [15]. We evaluated the two versions of our method (with and without "Planar Assumption"). The chosen parameters correspond to those determined by simulated annealing for uniform fog (Table I).

\begin{tabular}{|c|l|l|l|l|l|}
\hline Algorithm & Uniform & $\begin{array}{c}\text { Variable } \\
\beta\end{array}$ & $\begin{array}{c}\text { Variable } \\
I_{s}\end{array}$ & $\begin{array}{c}\text { Variable } \\
\beta \& I_{s}\end{array}$ & All types \\
\hline Nothing & $81.6 \pm 12.3$ & $78.7 \pm 12.3$ & $69.0 \pm 10.9$ & $66.4 \pm 10.8$ & $73.9 \pm 13.2$ \\
MSR [5] & $46.7 \pm 16.3$ & $86.4 \pm 24.7$ & $44.8 \pm 17.1$ & $83.7 \pm 24.9$ & $65.4 \pm 28.9$ \\
CLAHE [16] & $66.9 \pm 10.7$ & $64.5 \pm 9.7$ & $54.5 \pm 8.5$ & $54.6 \pm 7.8$ & $60.1 \pm 10.9$ \\
DCP [2] & $46.3 \pm 15.6$ & $46.9 \pm 17.0$ & $43.7 \pm 16.2$ & $44.1 \pm 17.5$ & $45.2 \pm 16.7$ \\
FSS [3] & $34.9 \pm 15.1$ & $40.9 \pm 13.5$ & $32.5 \pm 11.4$ & $36.5 \pm 10.3$ & $36.3 \pm 13.1$ \\
NBPC [15] & $50.8 \pm 11.5$ & $50.5 \pm 11.5$ & $38.5 \pm 9.0$ & $38.0 \pm 8.7$ & $44.5 \pm 12.1$ \\
NBPC+PA[15] & $31.1 \pm 10.2$ & $36.0 \pm 10.3$ & $26.7 \pm 5.1$ & $28.4 \pm 5.9$ & $30.6 \pm 8.9$ \\
Our & $30.9 \pm 11.1$ & $25.9 \pm 7.1$ & $23.5 \pm 6.1$ & $24.4 \pm 5.9$ & $26.1 \pm 8.3$ \\
Our + PA & $29.6 \pm 11.3$ & $24.0 \pm 7.7$ & $25.7 \pm 4.4$ & $25.2 \pm 6.2$ & $26.1 \pm 8.1$ \\
\hline
\end{tabular}

Table II: Comparison of restoration methods. Mean absolute difference between restored images and original images without fog, for the 7 compared algorithms and for 4 types of fog.

The obtained result clearly shows that our method outperforms all existing restoration approaches, regardless of the nature of the fog (homogeneous or inhomogeneous). The error decreases of at least a factor of $12 \%$ in comparison with the best available result.

It shows that using or not of the "planar assumption" gives equivalent results. The "planar assumption" provides better results when $I s$ is constant. However, the restoration of the road surface is degraded by the "planar assumption" when $I s$ varies. This comes from the fact that the model used for the restoration is not valid for this type of fog.

The method FSS in Table II is a variation of the "planar assumption approach" [3]. We can see the improvement that we have made to this method by combining it with our approach.

\section{Conclusion}

We have proposed a method for restoring the contrast of road images taken in fog. The originality of our method lies in the combination of a physical approach, based on Koschmieders model and a signals approach, based on local histogram equalization. The evaluation of our algorithm on $66 * 4$ synthetic images with various types of fog shows that our algorithm outperforms all existing restoration method.

To validate the choice of using or not the "planar assumption" in combination with our method, we plan to test the restoration on advanced driving assistance system.
Depending of the improvement of the system behavior, we will select the best configuration of the final restoration method.

\section{References}

[1] R. Fattal. Single image dehazing. ACM SIGGRAPH 2008, p. 1-9. 2008.

[2] K. He, J. Sun and X. Tang. Single image haze removal using dark channel prior. Computer Vision and Pattern Recognition, IEEE Computer Society Conference, p. 1956-1963. 2009.

[3] N. Hautière, J.-P. Tarel, and D. Aubert. Mitigation of Visibility Loss for Advanced Camera based Driver Assistances. In IEEE Transactions on Intelligent Transportation Systems, 11 (2): 474-484, 2010.

[4] N. Hautière, J.P. Tarel and D. Aubert. Free space detection for autonomous navigation in daytime foggy weather. Conference on Machine Vision Applications, p. 501-504. 2009.

[5] D. Jobson, Z. Rahman, and G. Woodell. A multiscale retinex for bridging the gap between color images and the human observation of scenes. IEEE Transactions on Image Processing, vol. 6, no. 7, pp. 965-976. 1997.

[6] K. Joung-Youn, K. Lee-Sup, H.S.-Ho. An advanced contrast enhancement using partially overlapped sub-block histogram equalization. IEEE Transactions on Circuits and Systems for Video Technology, pp. 475-484. 2001.

[7] J. Kopf, B. Neubert, B. Chen, M. Cohen, D.Cohen-Or, O. Deussen, M. Uyttendale, and D. Lischinski. Deep Photo: ModelBased Photograph Enhancement and Viewing, ACM Transactions on Graphics. 2008.

[8] L. Kratz and K. Nishino. Factorizing scene albedo and depth from a single foggy image. In ICCV, pages 17011708, 2009.

[9] W. Middleton. Vision through the atmosphere. University of Toronto Press. 1952.

[10] S.G Narasimhan and S.K. Nayar. Contrast Restoration of Weather Degraded Images. IEEE Transactions on Pattern Analysis and Machine Intelligence, vol. 25, p. 713 - 724. 2003.

[11] S.G. Narashiman and S. K. Nayar. Interactive deweathering of an image using physical model. IEEE Workshop on Color and Photometric Methods in Computer Vision. 2003.

[12] S. Shwartz, E. Namer, and Y. Schechner. Blind haze separation. IEEE Conf. Computer Vision and Pattern Recognition, 19841991, 2006.

[13] R. T. Tan. Visibility in bad weather from a single image. In Proc. of IEEE International Conference on Computer Vision and Pattern Recognition, pages 18, 2008.

[14] R.T. Tan, N Pettersson and L. Petersson. Visibility Enhancement for Roads with Foggy or Hazy Scenes. IEEE Intelligent Vehicles Symposium, p. 19-24. 2007.

[15] J.P. Tarel, N. Hautiere, A. Cord, D. Gruyer and H. Halmaoui. Improved visibility of road scene images under heterogeneous fog. Intelligent Vehicles Symposium. 2010.

[16] K. Zuiderveld. Contrast limited adaptive histogram equalization. Graphics gems IV. San Diego, USA: Academic Press Professional, Inc., pp. 474-485. 1994. 